\title{
UNIVERSAL SEROLOGIC REACTION IN LEPROSY
}

\author{
By Reuben L. Kahn \\ Department of Dermatology and Syphilology, and Serology Laboratory, University Hospilal, \\ University of Michigan, Ann Arbor, Michigan
}

It has long been known that the serum of certain leprosy patients gives positive reactions with tests for syphilis. These reactions have not been understood and have been interpreted as biologic "false positives." The reason for this interpretation undoubtedly lies in the widespread assumption that only in syphilis and in closely related diseases, such as yaws and bejel, are serum reactions with lipid antigen "true positives." We, ourselves, held this assumption for a number of years. In due time, cumulative experimental findings in this laboratory suggested that serum reactions with lipid antigen in the absence of syphilis are biologically significant. The question then arose whether the reactions might also be significant clinically in different diseases. In attempting to answer this question, a multiquantitative precipitation technique with lipid antigen was devised and applied to healthy individuals, to animals, and to patients with syphilis, yaws, leprosy, malaria, and tuberculosis. It was found that all individuals and all animals, whether in health or disease, gave positive precipitation reactions when tested with this technique. This finding led to the use of the term "universal serologic reaction." The basic characteristics of this reaction in health and in certain diseases will first be briefly reviewed. This step, it is believed, will help clarify the behavior of the reaction in leprosy.

Some Aspects of the Universal Serologic Reaction. The universal serologic reaction is a precipitation reaction elicited with lipid antigen. It exhibits qualitative and quantitative precipitation properties. The qualitative property determines the serologic pattern of the reaction. The basis of the pattern is the arrangement of precipitation in relation to given $\mathrm{NaCl}$ concentrations and to periods of incubation employed in the universal serologic technique. The quantitative property determines the degree of precipitation in the reaction, a factor which is independent of the serologic pattern.

Normal human beings give universal reactions of a characteristic serologic pattern. This pattern is believed to be the result of a precipitation reaction between natural auto-antibodies to lipids present in the serum and corresponding lipids present in the tissue extract antigen. These auto-antibodies are presumably formed in response to lipids, liberated in normal tissue wear and tear, which have antigenic properties.

The degree of precipitation in the universal reaction generally varies in different normal individuals, but is constant in the same individual. The variations in precipitation in different individuals are presumably due to different concentrations of auto-antibodies in the serum. These different concentrations are believed to be the result of differences in the liberation of antigenic lipids and in the capacities for antibody production. The relatively constant degree of precipitation in the same normal individual is 
presumably due to the relative constancy of the antibody concentration in the serum. This constancy is to be expected since the degree of lipid liberation and the capacity for antibody production are likely to be constant.

Judging from the universal reactions obtained in diseases studied thus far (syphilis, yaws, leprosy, malaria, and tuberculosis), two changes in the normal serologic pattern may be noted in a given disease: a qualitative change in the shape of the serologic pattern and a quantitative change in the intensity of precipitation. The change in the shape of the serologic pattern may be so distinctive as to result in a pattern entirely different from the normal serologic pattern, or the change may be of an apparently minor nature, resulting in a serologic pattern somewhat similar to the normal pattern.

The serologic pattern in a given disease appears to be unrelated to the degree of activity of the disease. The intensity of precipitation, however, is apparently closely related to the activity of the disease. When the activity is low, the intensity of precipitation is low. When the activity is increased, the intensity of precipitation is increased. An exception to this rule is noted when the activity of the disease is so marked as to overwhelm the patient. The intensity of precipitation, instead of being increased, is then actually decreased. As an illustration, the universal reaction in faradvanced, and miliary tuberculosis, in which precipitation generally drops to a low level, might be mentioned.

The serologic pattern of the universal reaction in a given disease is believed to be the result of particular lipids liberated in the tissue destructive processes of that disease. These lipids call forth homologous auto-antibodies which react with corresponding lipids present in the antigen. The fact that five different serologic patterns are obtained when sera from patients with syphilis, yaws, leprosy, malaria, and tuberculosis are examined with the lipid antigen suggests five different lipid antigen-antibody reactions. This fact further suggests that at least five different antigenic fractions are present in the antigen and that each fraction reacts with its homologous auto-antibodies present in the serum. Thus, lipid fraction " $A$ " reacts with the antibody in syphilitic serum; lipid fraction " $B$ " reacts with the antibody in lepromatous leprosy serum; lipid fraction " $\mathrm{C}$ " with the antibody in malaria serum, etc.

Universal Serologic Technique. ${ }^{1}$ In the universal serologic technique, the lipid antigen employed is similar to antigens used for tests for syphilis. The data herewith presented are based on a universal technique which consisted of seven quantitative set-ups, each of a different $\mathrm{NaCl}$ concentration. In the first set-up, the serial dilutions of serum were carried out with water; in the second, with 0.15 per cent $\mathrm{NaCl}$ solutions; in the third, with 0.6 ; in the fourth, with 0.9 ; in the fifth, with 1.2 ; in the sixth, with 1.8 , and, in the seventh, the serial dilutions of serum were carried out with 2.1 per cent. Fach serum was serially diluted with the appropriate diluent in ratios extending from 1:1 to 1:256. Standard Kahn antigen was employed in this study. The antigen suspension was prepared as for a Kahn test. The amount of suspension employed was $0.025 \mathrm{cc}$., to which was 
added $0.15 \mathrm{cc}$. of each serum dilution. The results of the seven quantitative set-ups were read after shaking the mixtures of serum dilutions with the antigen suspension for a period of three minutes, and also after four and 24 hours incubation at ice box $\left(5^{\circ}\right)$ temperature.

TABLE 1

Precipitation Readings of Seven Quantitative Set-ups of Different Salt CONCEnTRations

\begin{tabular}{c|c|c|c|c|c|c|c|c}
\hline \hline \multirow{2}{*}{ Serum dilutions } & \multicolumn{7}{|c}{$\%$ NaCl used in dihutions } \\
& 0 & .15 & .6 & .9 & 1.2 & 1.8 & 2.1 \\
\hline Undil. & 4 & 4 & 4 & - & 4 & 4 & 4 \\
$1: 2$ & 4 & 4 & 3 & - & \pm & 4 & 4 \\
$1: 4$ & 4 & 4 & \pm & - & \pm & 3 & 4 \\
$1: 8$ & 4 & 4 & \pm & - & \pm & - & 2 \\
$1: 16$ & 4 & 2 & - & - & - & - & - \\
$1: 256$ & 4 & - & - & - & - & - & - \\
\hline
\end{tabular}

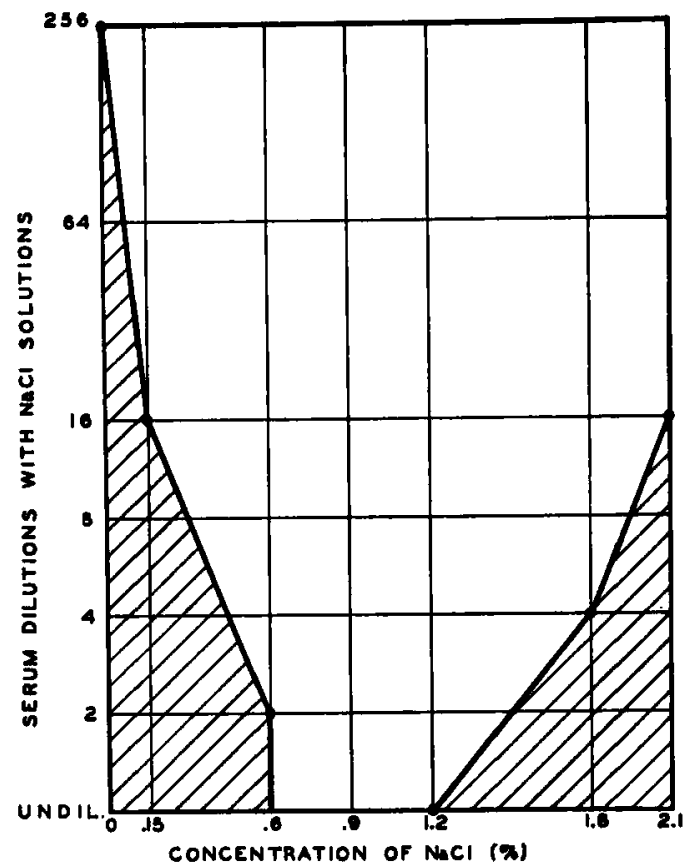

Figure 1. Precipitation readings of rabla 1 presented graphically.

An illustration of a universal reaction based on the seven quantitative set-ups, in which the precipitation results were read but once, is presented in TABLE 1 and is charted in the accompanying FIGURE 1. Most marked precipitation is noted in the quantitative set-ups of low (0 and 0.15 per cent) and of high (1.8 and 2.1 per cent) $\mathrm{NaCl}$ concentrations. Least 
REPEAT EXAMINATIONS - NORMAL INOIVIDUALS
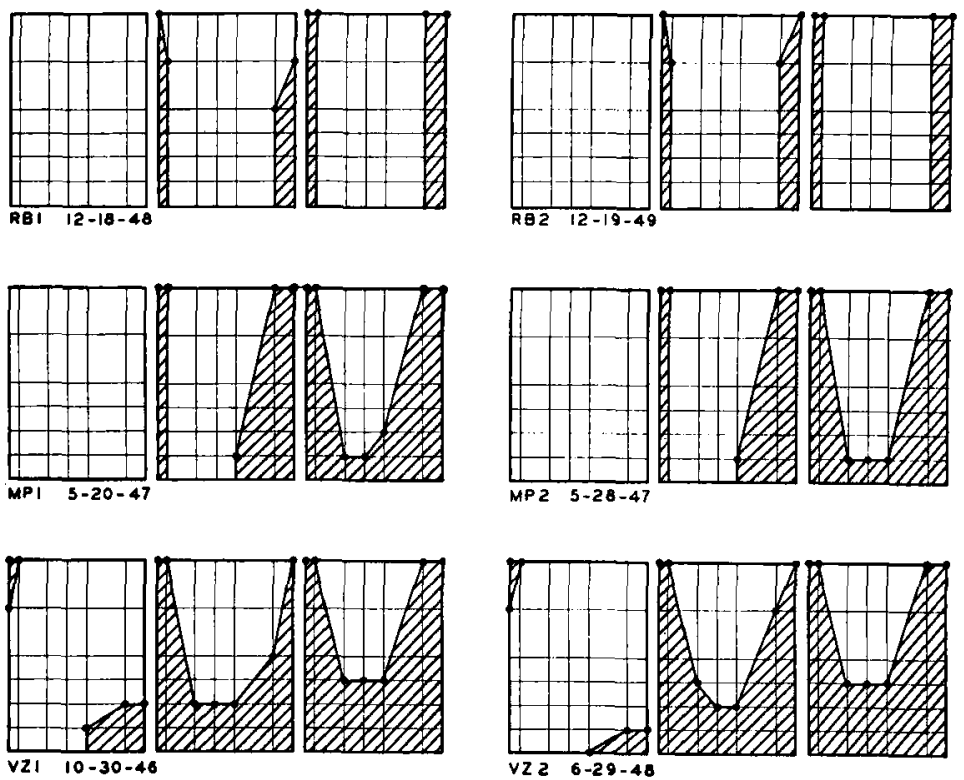

FIGURE 2. Illustrating the serologic pattern in health with different degrees of precipitation in different individuals and with constant precipitation in the same individual.

SYPHILIS
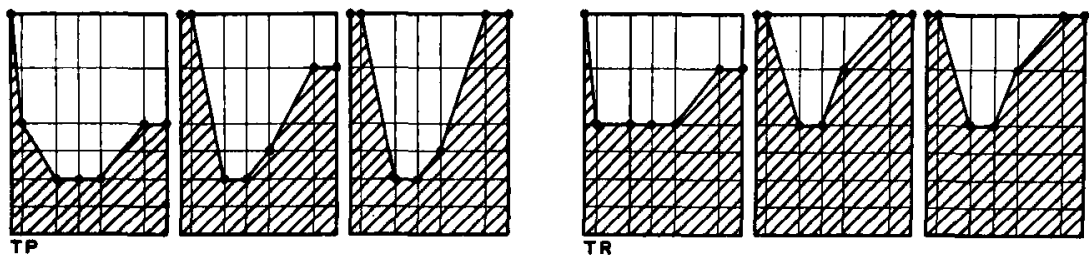

YAWS
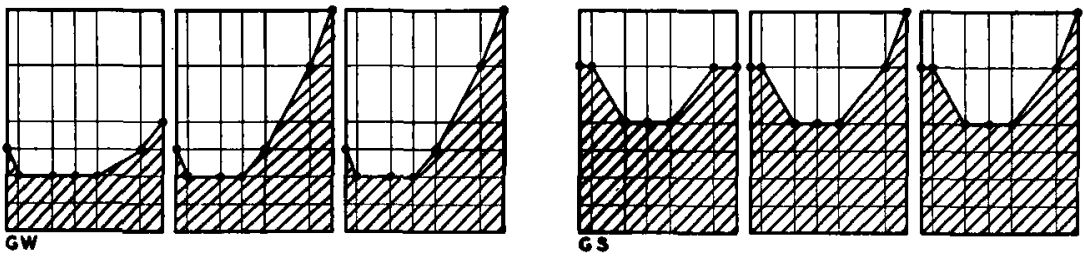

Figure 3. Illustrating the serologic patterns in syphilis and yaws.

precipitation is noted in the quantitative set-ups of moderate (0.6 and 1.2 per cent) $\mathrm{NaCl}$ concentrations, and no precipitation is noted in the quantitative set-up with 0.9 per cent $\mathrm{NaCl}$ solution.

The table is presented to demonstrate the basis of charting of the graph in FIGURE 1. The graph, in turn, is presented as a model of the graphs in 
FIGURES 2 to 4 . It is evident that each figure consists of three graphs, based on the readings of the precipitation results without incubation, and after four and 24 hours at cold temperature.

Universal Serologic Reactions in Normal Human Beings. Figure 2 presents universal reactions given by three normal human beings, RB, MP, and $\mathrm{VZ}$, based on repeat examinations. It is evident that $\mathrm{RB}$ shows least
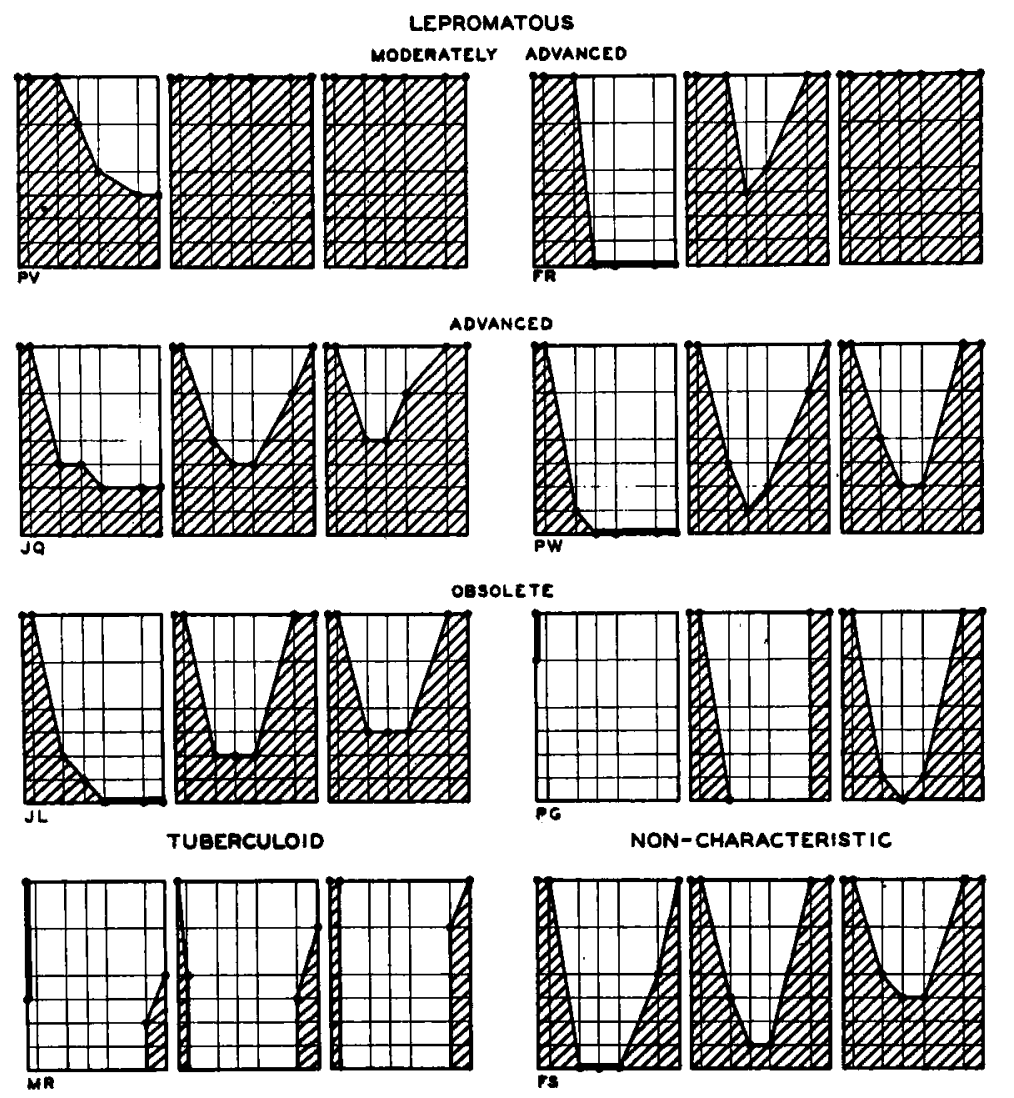

FIGORE 4. Illustrating the serologic patterns in leprosy.

precipitation, MP moderate precipitation, and VZ marked precipitation. In practice, it is far more common for universal reactions in normal individuals to exhibit a low degree of precipitation than moderate precipitation. Marked precipitation may be noted generally in less than five per cent of normal individuals. No precipitation is generally noted on reading the results without incubation. Precipitation then begins to be noted in the quantitative set-ups of low and high salt concentrations after four hours at cold temperature and it is often increased after 24 hours.

Two aspects of universal reactions in normal individuals are evident from FIGURE 2. First, the different shapes of the serologic pattern in different individuals. Second, the constancy of the shape of the serologic 
pattern in the same individual on repeated examination. It is thus seen that, while different individuals may show a serologic pattern of different degrees of precipitation, the same individual shows a pattern of a constant degree of precipitation.

This aspect is emphasized because in our earlier reports we considered tht universal reactions of $\mathrm{RB}, \mathrm{MP}$, and $\mathrm{VZ}$ as examples of three different serologic patterns. It seems to us now that they exhibit one serologic pattern of different degrees of precipitation. Actually, the three universal reactions exhibit the serologic pattern of normal individuals. Since the concentration of auto-antibodies is likely to vary in different individuals, quantitative differences in precipitation are to be expected.

Universal Serologic Reactions in Syphilis and Yaws. Figure 3, universal reactions of syphilis and yaws, is presented for several reasons. The chart offers an opportunity to differentiate: (1) the normal serologic pattern from the pattern of syphilis, (2) the pattern of syphilis from the one in yaws, and (3) these three patterns from the pattern in leprosy.

The serologic pattern in syphilis is characterized by the formation of precipitation without incubation, and by showing broadly the same contour of precipitation before and after incubation at cold temperature. The quantitative set-ups of low and of high $\mathrm{NaCl}$ concentrations often show some increase in precipitation on cold incubation. But the quantitative set-ups in the central zone of the graph, in the region of 0.9 per cent $\mathrm{NaCl}$ concentration, generally show little or no increase in precipitation on cold incubation.

In yaws, the quantitative set-ups of low salt concentration show relatively little precipitation compared with the corresponding set-ups in syphilis. As is evident also from the graphs of yaws in FIGURE 3, the degrees of precipitation in the quantitative set-ups of low salt concentration are not affected by cold incubation. Neither is the degree of precipitation in the central zone of the graphs affected by cold incubation. The yaws patients from whom serum specimens were obtained for these universal reactions have been treated. In this laboratory, untreated yaws patients have given somewhat irregular serologic patterns, due presumably to intercurrent diseases.

Universal Serologic Reactions in Leprosy. A distinct leprosy pattern has thus far been obtained only in the lepromatous form. As is evident from FIGURE 4, precipitation in this form of leprosy is noted without incubation of the quantitative set-ups, as is true in syphilis and yaws. The precipitation pattern in this form of leprosy, however, is entirely different from the patterns in syphilis and yaws.

In lepromatous leprosy, precipitation without incubation of the quantitative set-ups is most marked in the low salt concentrations and decreases in intensity as the salt concentrations are increased. This shape of the first graph of the serologic pattern is believed to be typical for lepromatous leprosy and is noted in the patterns of PV, JQ, JL, FR, and PW of FIGURE 4. Incubation at cold temperature changes this shape. But this change is not a characteristic of lepromatous leprosy. As is evident from the report by Thiers Pinto and Zeo, treated patients with lepromatous leprosy 
often maintain the typical shape of the first graph of the serologic pattern after cold incubation.

The degree of precipitation in the universal reaction in lepromatous leprosy is apparently related to the degree of activity of the disease. In the moderately advanced form of the disease, the patient is apparently capable of producing the highest antibody concentration. The tissue destructive processes are relatively marked. The lipid liberation and the corresponding antibody production, therefore, are also relatively marked.

In advanced lepromatous leprosy, the antibody concentration is low, as is true in far-advanced tuberculosis. Two factors probably interfere with antibody production. The unusually marked tissue destructive processes undoubtedly have a debilitating effect on the patient. These destructive processes may also liberate antigenic lipids in sufficient excess as to reduce antibody production. The result is reduced precipitation in advanced lepromatous leprosy as indicated in Graphs JQ and PW of FIGURE 4.

When lepromatous leprosy is in a quiescent or obsolete form, we would also expect reduced precipitation in the universal reaction. The tissue destructive processes being at a low level, the liberation of antigenic lipids and the production of auto-antibodies are similarly at a low level.

In the case of tuberculoid and non-characteristic leprosy, we have thus far not observed any distinctive serologic pattern, and the degree of precipitation is generally low. It is often lower in tuberculoid than in noncharacteristic leprosy, due presumably to reduced activity in tuberculoid leprosy.

Leprosy patients often have intercurrent diseases that are likely to affect both the serologic pattern and the intensity of precipitation in the universal reaction. Chief among these diseases is syphilis, which has its own serologic pattern as well as its own relationship between the intensity of precipitation and the degree of activity of the disease. The universal reaction in lepromatous leprosy, therefore, does not always show a typical serologic pattern nor the expected relationship between the intensity of precipitation and the degree of activity of the disease. Treatment of the intercurrent disease might be expected to change this situation.

Single universal reactions in patients with leprosy may differentiate lepromatous from other forms of leprosy, otherwise it may give relatively little serologic information, as is true of single quantitative serologic reactions in patients with syphilis. For example, a low serologic titer in syphilis might mean that the disease (1) is in the early primary stage, (2) is in a late stage, approaching seronegativity following therapy, (3) is in a state of low activity without regard to the stage of the disease, or (4) is in a precocious malignant state, a rare state in which the disease is overwhelmingly active. As is well known, repeated quantitative serologic studies in syphilis, in combination with clinical studies, are necessary for the correct interpretation of the serologic results. The same applies to universal serologic studies in leprosy.

Indeed, a great deal of information has accumulated on serologic reactions with lipid antigen in syphilis in relation to clinical findings, both before and after treatment. This information has accumulated because physicians 
have been taking blood specimens for serologic examinations with relative frequency and patients with syphilis have grown accustomed to give such specimens readily. If the value and limitations of the universal reaction in leprosy are to be established, the serologic examinations similarly must be made at intervals and the results correlated with clinical findings.

Perhaps the greatest potential value of the universal reaction in leprosy lies in its application as a guide to therapy in this disease. Precipitation in the universal reaction should be reduced with improvement, as is true with serologic reactions following therapy in syphilis. An exception will be noted when precipitation is low, not because of low activity of the disease but because of excessive activity, which results in a reduction of the capacity for antibody production. Then, as the excessive activity of the disease is halted by therapy, antibody production and resulting precipitation should increase. If the therapy should cause the activity to drop to a low level, then precipitation should also drop to a low level.

This report is in the nature of an abstract of a more comprehensive discussion of the universal serologic reaction in leprosy, ${ }^{2}$ and it is presented at this time with the hope that it will stimulate workers to undertake studies of the universal reaction in leprosy in areas where the disease is endemic.*

\section{Conclusions}

1. The serologic pattern of the universal reaction in lepromatous leprosy is distinctive and different from the pattern noted in certain other diseases (syphilis, yaws, malaria, and tuberculosis). In practice, not all patients with lepromatous leprosy show a distinctive pattern, due, it is believed, to intercurrent diseases, especially syphilis.

2. No distinctive serologic pattern has thus far been observed in tuberculoid and non-characteristic leprosy.

3. The intensity of precipitation in the universal reaction in patients with lepromatous leprosy is related to the degree of activity of the disease. The intensity of precipitation is low when the activity is at a low level, and it is increased when the activity is moderately increased. The intensity of precipitation is reduced, however, when the activity is markedly increased.

4. The universal reaction gives promise to be of value in leprosy, especially as a serologic guide to therapy, provided the universal results are obtained from time to time and correlated with clinical findings.

\section{References}

1. KAHN, R. L. 1949. Universal Serologic Reaction with Lipid Antigen. I. In Normal Persons. II. In Animals (With Betty J. Baribeau). III. In Syphilis (With Elizabeth B. McDermott). IV. In Lepromatous Leprosy (With Betty J. Baribeau and Flora T. Villalon). V. In Malaria. VI. In Tuberculosis. Am. Jr. Clin. Path. 19: 347, $361,401,408,414,419$.

2. KAHN, R. L. 1951. An Introduction to Universal Serologic Reaction in Health and Disease. The Commonwealth Fund. New York; 1950. Serology with Lipid Antigen, with special reference to Kahn and universal reactions. Williams and Wilkins. Baltimore, Md.

* The writer is indebted to Doctor Alberto Oteiza and Doctor Flora T. Villalon, of Havana, and to Doctor George L. Fite, of the National Institute of Health, Bethesda, Md, for furmishing the blood specimens with clinical histories from leprosy patients employed in this study. 